\title{
Information to the Eye of the Beholder: Data Visualization for Many-Objective Optimization
}

\author{
Ivan Reinaldo Meneghini*, Roozbeh H. Koochaksaraei*, Frederico G. Guimarães* and Antonio Gaspar-Cunha ${ }^{\dagger}$ \\ ${ }^{*}$ Machine Intelligence and Data Science (MINDS) Laboratory, Universidade Federal de Minas Gerais, Belo Horizonte, Brazil \\ Email: fredericoguimaraes@ufmg.br \\ ${ }^{\dagger}$ Institute for Polymers and Composites, University of Minho, 4800-058 Guimarães, Portugal \\ Email: agc@dep.uminho.pt
}

\begin{abstract}
Lorem ipsum dolor sit amet, consectetur adipiscing elit. Proin mattis, massa at dignissim luctus, sem sapien lobortis massa, a ullamcorper urna ipsum quis lorem. Suspendisse potenti. Ut venenatis condimentum fringilla. Nulla elementum elit lectus. Integer ut bibendum orci, sit amet eleifend arcu. Phasellus nec velit vulputate, facilisis lacus nec, facilisis tortor. Morbi eget erat justo. Quisque placerat ultrices ex, sit amet accumsan neque tempor ac. Aenean vel magna lobortis felis rutrum ultrices. Sed vestibulum malesuada vestibulum. Phasellus egestas elementum dolor, nec maximus ante. Duis porttitor, erat sit amet molestie mattis, massa ipsum vulputate est, vitae maximus augue ipsum at nisi. Donec ut est iaculis, dignissim erat et, ultrices eros. Aenean cursus, elit id pellentesque molestie, est erat viverra ligula, sit.
\end{abstract}

\section{INTRODUCTION}

Multiple and often conflicting objectives naturally arise in many practical problems in engineering, leading to the socalled multi-objective optimization problems [1]. The solutions to multi-objective optimization problems are characterized by the trade-off relationship among the objectives. This set of solutions is called the set of Pareto-optimal solutions and its corresponding image in the space of objectives is named the Pareto front. In general, finding the complete and exact set of Pareto-optimal solutions is a hard task, therefore many heuristic methods have been developed by the optimization community to find approximations of this set, especially multiobjective evolutionary algorithms (MOEA) [2, 3].

When there are few objectives to be considered, evolutionary algorithms are usually suitable to find high quality approximations of the Pareto front. As the number of objectives grow, we reach the field of many-objective optimization problems (MaOPs) $[4,5]$. The optimization of those problems becomes harder as most solutions become incomparable in relation to Pareto dominance. This border is not actually pecisely defined but it has practical meaning, based on empirical studies about the downgrading performance of most multi-objective algorithms when the number of objectives increase. MOEA have been successful and specialized to low dimensional multiobjective problems, but their performance degrades with the increasing number of objectives, as already discussed in many different studies, see for instance [6].

The visualization gap is one of the important challenges that are posed by MaOPs, as identified in a number of studies $[7,8]$. It is an obstacle to the interpretation of results, qualitative assessment of the algorithms and in the analysis of MOEA for MaOPs. An intuitive and high quality visualization can enable the decision-maker to recognize characteristics of the problem, realize the underlying trade-off, distribution of solutions, among other features [9]. Over the years, many reseachers have provided interesting visualization methods for MaOPs. As evidence of the growing relevance of this issue, it is notable that the last conferences of IEEE CEC and ACM GECCO have hosted tutorials and special sessions dedicated to the topic of visualization in MaOP.

Perhaps the most common approach for data visualization in MaOPs is the Parallel Coordinates, given in [10]. An interesting discussion on how to interpret results using Parallel Coordinates can be found in [11]. The Radar chart (also known as spider chart or polar chart) is also often used for visualization [7]. It is simply a polar representation of the parallel coordinates. Pryke et al. [12] presented a method based on Heatmaps, for visualizing the objective and design space simultaneously. Another interesting approach is RadViz (Radial Coordinate Visualization) [13, 14], which is a data visualization tool inspired from physics. RadViz is a display technique that places dimensional anchors (dimensions) around the perimeter of a circle. Spring constants are used to represent relational values among points - one end of a spring is attached to a dimensional anchor, the other is attached to a data point. Each data point is displayed at the point where the sum of all spring forces equals zero. More recently, $\mathrm{He}$ and Yen [8] presented a visualization method based on mapping the high dimensional space into polar coordinate plot while preserving the important characteristics of objective spaces such as Pareto relationship, shape and location of the Pareto front and the distribution of individuals. According to the observed information from this tool, a new performance metric named polar-metric has been proposed.

The method of Aggregation Trees [15, 7] was another important step in visualization in MaOPs, allowing a greater understanding of the problem. This method is based on the sequential aggregation of objectives, which is visually represented into a tree, based on a measure of conflict between pairs of (groups of) objectives. The method allows the visualization of a hierarchy for aggregation of the objectives, with possibility to create new constraints for the problem or reduce 
the number of objectives in a further analysis [16]. Later, in our previous work, we produced a form of interactive circular visualization for inspecting the relationship among objectives in a chord diagram [17], in order to assist understanding of the problem by the decision-maker. In an extension of this work, we developed a new visualization tool that follows the circular design layout, mapping the solutions from high-dimensional objective space into a $2 \mathrm{D}$ form of scattering that is based on norm and angle information [18].

In this paper, we present a further development on this visualization tool, integrating the chord diagram, the parallel coordinates and the norm and angle mapping into the same figure. The new visualization tool was developed in $\mathrm{R}$ language and it will be available to the scientific community under GNU license. It also follows the circular design layout, with layers or tracks showing different levels of information. In terms of data visualization techniques, the circular layout is an interesting way to present large volume of data, because in addition to being aesthetical to most people, it is an elegant and easy way to illustrate relations among elements of the data such as oneto-many, many-to-one and many-to-many. In the new version developed, it is possible to visualize the format and distribution of the Pareto front, the harmony and conflict between the objectives and also the complementary information of the parallel coordinates, all in the same visualization. With this work, we try to fill the visualization gap and bring information to the eye of the decision-maker and the optimizer, with an intuitive overview of the obtained results.

In order to illustrate the integrated visualization tool, some experiments were performed using the Benchmark Functions proposed for the IEEE-CEC 2018 Competition on ManyObjective Optimization $[19,20]$. We use the tool to visualize the results obtained by the algorithms NSGA-III [21], HypE [22], RVEA [23], MOEA/DD [24] and PICEA-g [25], using the PlatEMO MATLAB platform [26], with the same parameter settings of the competition. The results on the Benchmark Problems show the importance of the qualitative analysis of the data. The experiments show how visualization can help interpretation of the results and identification of strengths and drawbacks of MOEA.

\section{Grouped Visualization In CiRcular LAYOUT}

\section{A. Overview}

The proposed visualization tool brings together in a single figure three visualization methods: the Parallel Coordinates [11], the Chord Diagram [17] and the Angular Mapping [18]. Using a circular design layout provided by the Circlize package [27] for R language, this method enables the spatial location of points in high dimensional spaces, the visualization of harmony/conflict among objectives, as well as the comparison of approximation sets provided by different algorithms.

The circular design layout is composed of sectors and tracks. Each sector is associated with an axis in objective space (considering the orthogonal system of rectangular coordinates to represent the original objective space) and each track corresponds to a different method of visualizing data. The input data is matrix of size $N \times M$ containing a set of $N$ points in the $M$-dimensional objective space, corresponding to an approximation of the Pareto front. These points are then divided among the objectives according to the angular distance to the coordinate axes. Each point is associated with the sector corresponding to the nearest coordinate axis. Figure 1 illustrates the elements of a sector: track A: Chord Diagram; track B: Parallel Coordinates; and track C: Angular Mapping. The objective index is indicated at the top of the sector (D). The three visualization methods of each track are discussed in more detail in the following sections.

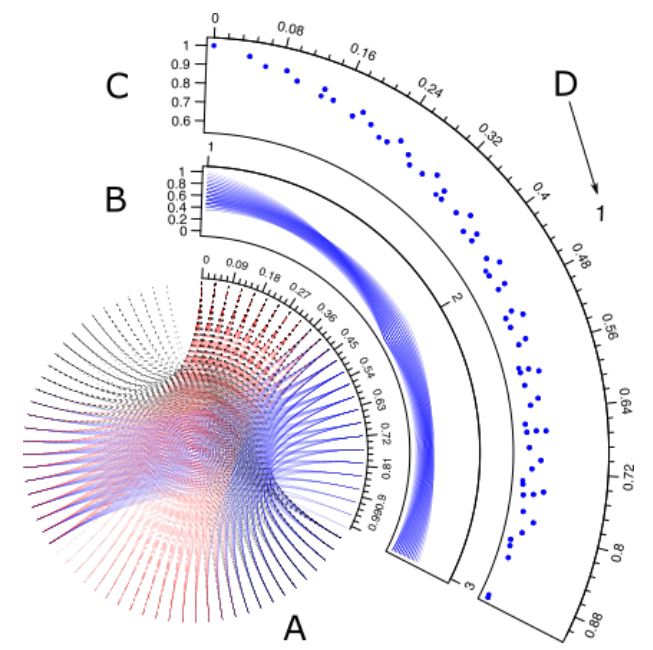

Fig. 1: A: Chord Diagram; B: Parallel Coordinates; C: Angular Mapping; D: index of the objective function.

This method was implemented in $\mathrm{R}$ using the Circlize package [27], which in turn is based on the ideas of Circos [28]. High definition images as well as the R-script are available for download from the MINDS Lab website.

\section{B. Chord Diagram}

The first track of the circular layout is used to display the chord diagram for MaOPs [18]. The Chord Diagram is a circular approach to display vectors from an $M$-dimensional space into a two-dimensional chart. In the Chord Diagram, each arc represents one objective function of the problem. The range of each objective in each arc is represented in proportion along the arc length, with all the objectives of all sections forming the circle. The range for each objective is shown from the lower bound to the upper bound in clockwise direction. The lower and upper bound of each objective can be obtained directly from the data or set by the user. By default, the range is normalized to $[0,1]$. Each point in the $M$-dimensional objective space is displayed by Bézier curves connecting the corresponding values in each arc (each coordinate).

\section{Parallel Coordinates}

Parallel Coordinates is a usual technique for representing high dimensional data [11]. In this method, the data are shown in a two-dimensional chart, where each category corresponds to a point on the horizontal axis and the values in each 
category are represented on the vertical axis. In this way, each point in $M$-dimensional space is represented by means of a polyline with $M-1$ segments passing through each of these coordinates in the plane. The sequence of the data on the horizontal axis generally follows the (usually arbitrary) order established in the vector. However, for a better visualization of the correlations between the objectives, this order can be changed. The use of this method is not suitable for data that are on very different scales from one another, therefore normalization of data is often used. In addition, the representation of many points by this method produces a drawing with many lines, which is more difficult to analyze. It is important to mention that only those points associated with the objective and section are displayed in the track corresponding to the Parallel Coordinates. Moreover, since we embed this visualization into a section of the circle layout, it bends the original Parallel Coordinates into the corresponding track of the sector.

\section{Angular Mapping}

The Angular Mapping proposed in [18] uses three features to map a point $\mathbf{y}$ in the $M$-dimensional objective space into a two-dimensional chart. The first feature is the $p$-norm, usually $p=2$, of the vector i.e. $\rho=\|\mathbf{y}\|_{p}$. The second feature is the smallest angle $\theta$ between the vector and the vectors $\mathbf{e}_{1}, \mathbf{e}_{2}, \ldots, \mathbf{e}_{M}$ of the canonical base, given by:

$$
\begin{gathered}
\alpha_{i}=\arccos \left(\frac{y_{i}}{\rho}\right), \quad i=1, \ldots, M \\
\theta=\min _{i} \alpha_{i}
\end{gathered}
$$

The third feature is the index $j$ for which the smallest angle occurs:

$$
j=\arg \min _{i} \alpha_{i}
$$

The index $j$ determines the division of the input data in the sectors, that is, sector $j$ will display only those points for which the third feature is equal to $j$. In this way, the points represented by the parallel coordinates and the angular mapping in a given sector are those that have the smallest angle with the axis representing the objective function of that sector. In the $M$-dimensional space, the maximum value for the angle is the one between the hyper diagonal $h=(1,1, \ldots, 1)$ and any canonical base vector $\mathbf{e}_{i}=(0, \ldots, 1, \ldots, 0)$, which can be calculated as $\theta_{\max }=\arccos (1 / \sqrt{M})$ (in radians). With these three values, it is possible to obtain an estimate of the spatial location of the vector in space, as well as its relative position in relation to other vectors. Unfortunately, some loss of information is inevitable in this mapping, since different vectors may have the same values of $\rho, \theta, j$. The Angular Mapping is displayed on track $\mathrm{C}$, with the vertical scale (norm values) on the left and the horizontal scale (angles ranging from 0 to $\left.\theta_{\max }\right)$ on the top.

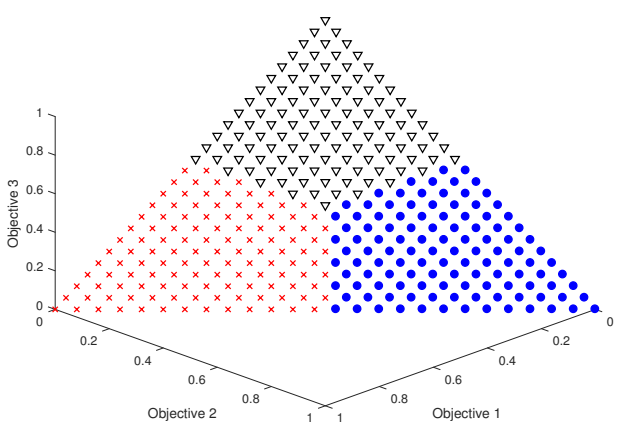

(a) Points in a flat surface using scatter plot.

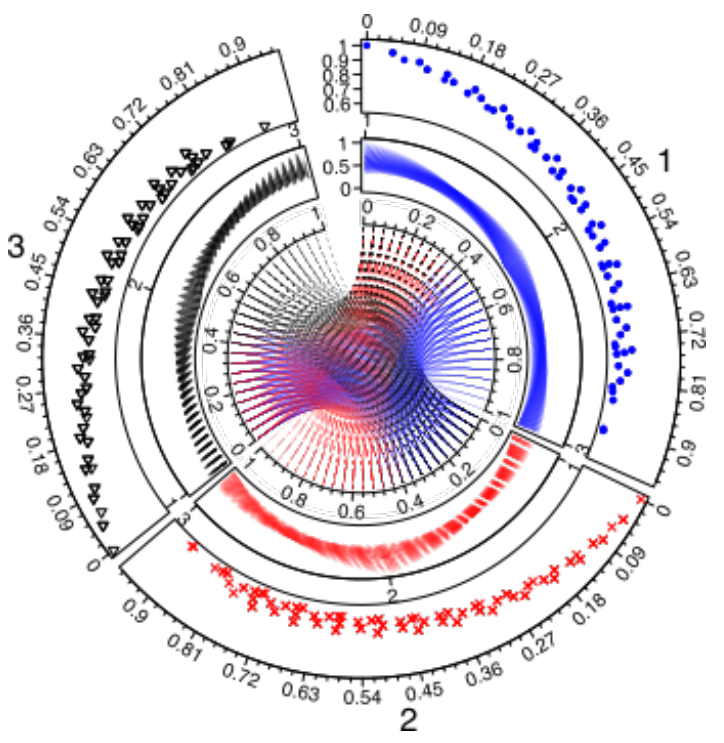

(b) Visualization of the points in the integrated tool.

Fig. 2: Classification of points by angular proximity to the coordinate axes.

\section{E. Examples}

In this section we present some examples in order to better illustrate the proposed visualization tool. In Fig. 2a, we plot 350 points evenly distributed on a flat surface in the first orthant of the three-dimensional space using scatter plot. The points are classified according to their angular proximity to each of the coordinate axes ${ }^{1}$, identified as objective functions. In this way, the red dots (marked as $*$ ) are closer to objective 1 , the blue dots (marked as + ) are closer to objective 2 and the black dots (marked as $\times$ ) are closer to objective 3 . Figure $2 \mathrm{~b}$ shows the visualization of these points using the proposed integrated visualization tool, preserving the color association.

In Fig. 2a, considering the black dots, objectives 1 and 2 vary in the interval $(0.0 .5)$ while the objective 3 varies in the interval $(0.5,1)$. This fact can be clearly seen in Fig. $2 b$ on sector 3, in tracks A and B (chord diagram and parallel

\footnotetext{
${ }^{1}$ Note that the smallest angle necessarily corresponds to the highest objective value.
} 


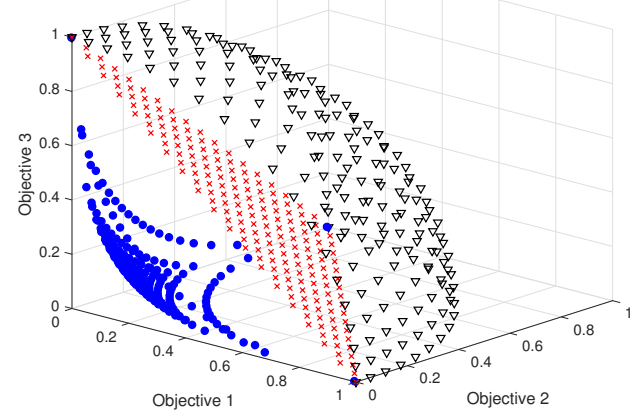

(a) Scatter plot representation.

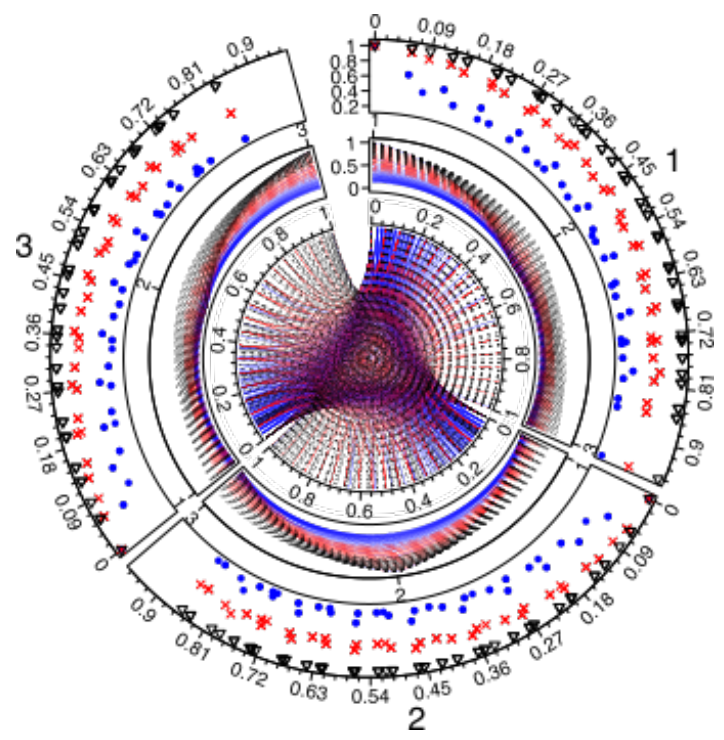

(b) Visualization of the points in the integrated tool.

Fig. 3: Surfaces with different norm in $\mathbb{R}^{3}$

coordinates, respectively). The spatial distribution of these points can be observed on track C. Since the points lie in a plane, the individual with the highest norm has the smallest angle and the points with smaller norm are closer to the maximum angle (they are located in the center of the plane, see Fig. 2a). Due to the symmetry of the figure, these same observations can be made for the red and blue dots.

Figure 3a shows a scatter plot of points distributed on surfaces of constant p-norm ( $p$ equal to $0.5,1$ and 2). The corresponding representation of these points in the integrated visualization tool is shown in Fig. 3b. Note that the relative position of the three surfaces can be easily seen on track $\mathrm{C}$ of Fig. 3b.

\section{RESULTS AND DISCUSSION}

In order to explore the possibilities of using the integrated visualization tool to aid the qualitative analysis of the obtained solutions, some experiments were carried out using the Benchmark Functions proposed for IEEE-CEC 2018 Competition on Many-Objective Optimization [19, 20]. For each problem in the Benchmark Functions, we performed a single run of the algorithms NSGA-III [21], Hype [22], RVEA [23], MOEA/DD [24] and PICEA-g [25], using the PlatEMO MATLAB platform [26], with the same parameter settings of the IEEECEC 2018 Competition on Many-Objective Optimization. To measure the quality of the approximation sets returned by the algorithms, we used the inverted generational distance (IGD) [29] and the hypervolume (HV) [30] performance indicators, which are shown in the Table I. Of course, the comparison of the results in Table I should be done with care and reservation, since any meaningful comparison of these algorithms using performance indicators should require multiple runs and statistical analysis. We will leave this task to the organizers of the competition. Our intention here is to contrast the visualization of these results with the quantitative comparison suggested by these perofrmance indicators, this is why we utilize results from a single run.

Due to space constraints, we cannot present the results on all the Benchmark Functions proposed for IEEE-CEC 2018 Competition on Many-Objective Optimization, therefore the most interesting cases according to the judgement of the authors are presented here. All the results and figures will be available as supplementary material to this paper. Given the space limitation, we have selected the functions $\mathrm{MaF} 1, \mathrm{MaF} 2$, MaF5, MaF7 and MaF10.

Next we present the visualization of the results for the problems MaF1, MaF5, MaF7 and MaF10 using the proposed integrated tool. In order to obtain a better visualization of the data, all the true Pareto fronts were normalized to the interval $[0,1]$. In this way, the obtained solutions by the algorithms preserve their relative position in relation to the true Pareto front. In tracks A and B only the solution obtained is presented. Track $\mathrm{C}$ shows the obtained solutions (blue dots) and the Pareto front (black dots).

\section{A. MaF1 Benchmark Problem}

This problem has a linear plane with inverted Pareto front. In the objective space, all solutions must be in the $[0,1]$ interval. Figure 4 shows the obtained solutions for this problem, with 5 objectives. The Pareto Front representation of this problem has a well-defined format, consisting of four sections of aligned points: a larger section, an intermediate section and two small sections. Only the Algorithm PICEA-g, see Fig. 4e, obtained solutions close to the medium and small sections. The algorithms NSGAIII and HypE. Fig. 4a and Fig. 4b, present points close only to the main section. The solutions obtained by MOEA/DD, see Fig. 4d, are highly concentrated in small regions and there are few solutions close to objective 5 . This poor performance is probably due to the generation of the weight vectors and replacement strategy of the version of MOEA/DD implemented in PlatEMO. Nonetheless, this algorithm was able to obtain solutions very close to the Pareto front of the problem, although badly distributed. The algorithm RVEA, Fig. 4c, presents few solutions. It is also possible to see some solutions in sector 3 and solutions in sector 5 with unsatisfactory convergence. It suggests that RVEA was 
TABLE I: IGD and HV metrics for the problems with 5, 10 and 15 objectives.

\begin{tabular}{|c|c|c|c|c|c|c|c|c|c|c|}
\hline & \multicolumn{2}{|c|}{ MaF1 } & \multicolumn{2}{|c|}{$\mathrm{MaF} 2$} & \multicolumn{2}{|c|}{ MaF5 } & \multicolumn{2}{|c|}{ MaF7 } & \multicolumn{2}{|c|}{ MaF10 } \\
\hline & IGD & $\mathrm{HV}$ & IGD & $\mathrm{HV}$ & IGD & $\mathrm{HV}$ & IGD & $\mathrm{HV}$ & IGD & $\mathrm{HV}$ \\
\hline \multicolumn{11}{|c|}{5 objectives } \\
\hline NSGAIII & 0.1865 & 0.0101 & 0.1134 & 0.0505 & 1.9698 & $4.2871 \mathrm{e}+04$ & 0.2824 & 2.2729 & 0.3961 & $6.0417 \mathrm{e}+03$ \\
\hline HypE & 0.2222 & 0.0071 & 0.1389 & 0.0552 & & & 1.4929 & 1.8265 & 1.6333 & $5.9083 e+03$ \\
\hline RVEA & 0.2848 & 0.0053 & 0.1199 & 0.0463 & 1.9693 & $4.2892 \mathrm{e}+04$ & 0.4855 & 1.9416 & 0.3675 & $6.0430 \mathrm{e}+03$ \\
\hline MOEA/DD & 0.2104 & 0.0092 & 0.1211 & 0.0451 & 3.9302 & $3.6174 \mathrm{e}+04$ & 0.4278 & 1.7530 & 0.7910 & $5.8907 \mathrm{e}+03$ \\
\hline PICEA-g & 0.1002 & 0.0206 & 0.0878 & 0.0549 & 1.5970 & $4.2180 \mathrm{e}+04$ & 0.5864 & 2.2232 & 0.3815 & $6.0427 \mathrm{e}+03$ \\
\hline \multicolumn{11}{|c|}{10 objectives } \\
\hline NSGAIII & 0.2613 & $1.1309 \mathrm{e}-06$ & 0.3201 & 0.0081 & 87.8409 & $9.0409 e+16$ & 1.1922 & 2.3349 & 1.1839 & $8.6667 \mathrm{e}+09$ \\
\hline HypE & 0.7040 & 0 & 0.5477 & 0.0091 & 136.0161 & $7.3166 e+16$ & 4.2172 & 2.1315 & & \\
\hline RVEA & 0.6475 & $1.1262 \mathrm{e}-08$ & 0.2310 & 0.0066 & 100.8695 & $8.9850 \mathrm{e}+16$ & 2.5231 & 2.1879 & 1.0398 & $8.6636 \mathrm{e}+09$ \\
\hline MOEA/DD & 0.3439 & $6.4020 \mathrm{e}-07$ & 0.1759 & 0.0068 & 283.1526 & $5.1348 \mathrm{e}+16$ & 2.8262 & $6.2928 \mathrm{e}-04$ & 1.8960 & $8.6089 \mathrm{e}+09$ \\
\hline PICEA-g & 0.2196 & $1.6355 \mathrm{e}-06$ & 0.2981 & 0.0085 & 56.5616 & $8.3100 \mathrm{e}+16$ & 4.7664 & 2.2025 & 2.5639 & $8.6666 \mathrm{e}+09$ \\
\hline \multicolumn{11}{|c|}{15 Objectives } \\
\hline NSGAIII & 0.3161 & $2.7216 \mathrm{e}-11$ & 0.2058 & $5.0942 \mathrm{e}-05$ & $2.5913 \mathrm{e}+03$ & $5.5045 e+36$ & 4.3990 & 2.1798 & 1.7279 & $1.3914 \mathrm{e}+17$ \\
\hline HypE & 0.5862 & 0 & 0.6185 & $8.2382 \mathrm{e}-05$ & & & 9.9815 & 1.9820 & 3.1511 & $1.3908 \mathrm{e}+17$ \\
\hline RVEA & 0.6276 & $8.4177 \mathrm{e}-14$ & 0.8551 & $1.7687 \mathrm{e}-05$ & $5.4979 \mathrm{e}+03$ & $5.2601 \mathrm{e}+36$ & 5.0452 & 2.0582 & 2.7017 & $1.3909 \mathrm{e}+17$ \\
\hline MOEA/DD & 0.4877 & $1.2558 \mathrm{e}-12$ & 0.4244 & $3.5341 \mathrm{e}-05$ & $7.3084 \mathrm{e}+03$ & $2.9503 \mathrm{e}+36$ & 3.1759 & $8.7923 \mathrm{e}-06$ & 2.4108 & $1.3844 \mathrm{e}+17$ \\
\hline PICEA-g & 0.2663 & 0 & 0.4131 & $6.4692 \mathrm{e}-05$ & $1.8505 \mathrm{e}+03$ & $4.8646 e+36$ & 10.5846 & 2.0319 & 2.8856 & $1.3914 \mathrm{e}+17$ \\
\hline
\end{tabular}

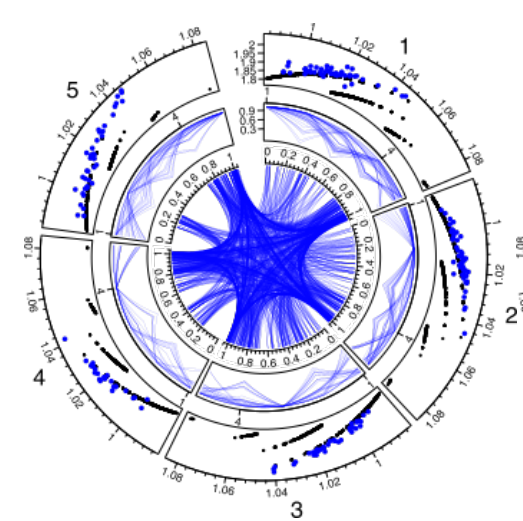

(a) MaF1 on NSGAIII

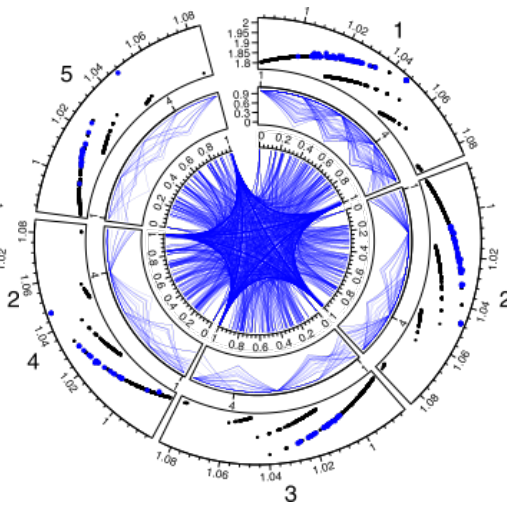

(b) MaF1 on HypE

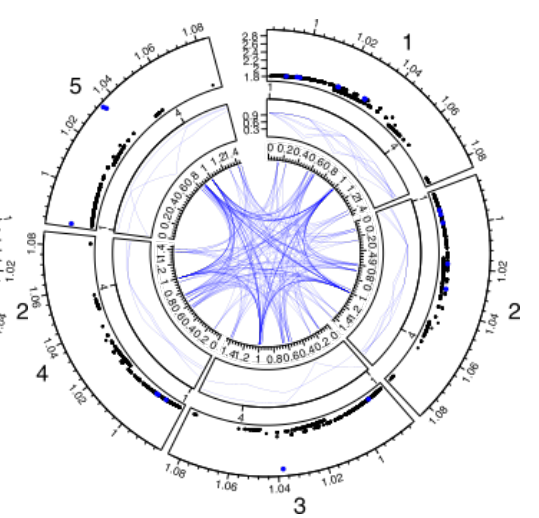

(c) MaF1 on RVEA

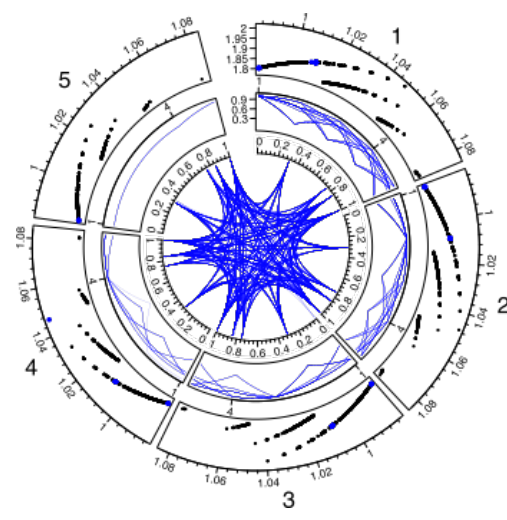

(d) MaF1 on MOEA/DD

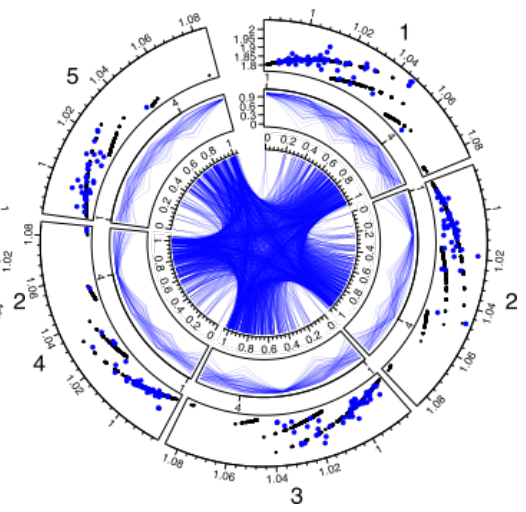

(e) MaF1 on PICEA-g

Fig. 4: MaF1 with 5 objectives

not able to eliminate these dominance resistant solutions in this particular run. Although they present similar metrics, the qualitative analysis of the figures in these particular runs indicates a clear superiority of the MOEA/DD algorithm when compared to the RVEA algorithm, in a hypothetical situation where these were the only choices. In these specific runs, NSGAIII and PICEA-g achieved better values of IGD and HV. This superiority is also clear in the visual comparison of the results. 


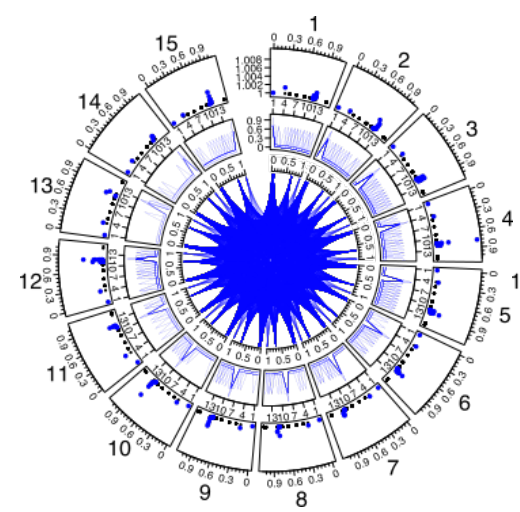

(a) MaF5 on NSGAIII

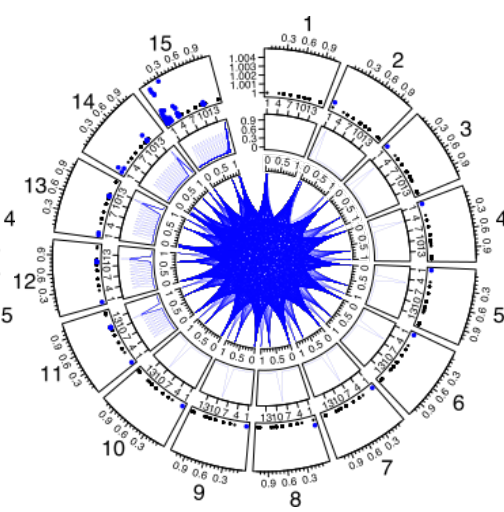

(b) MaF5 on RVEA

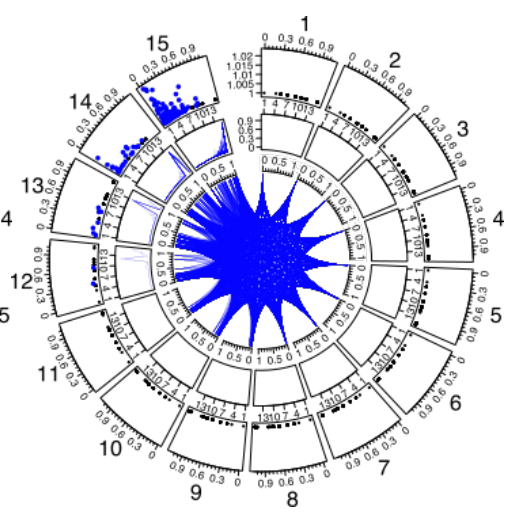

(c) MaF5 on MOEA/DD

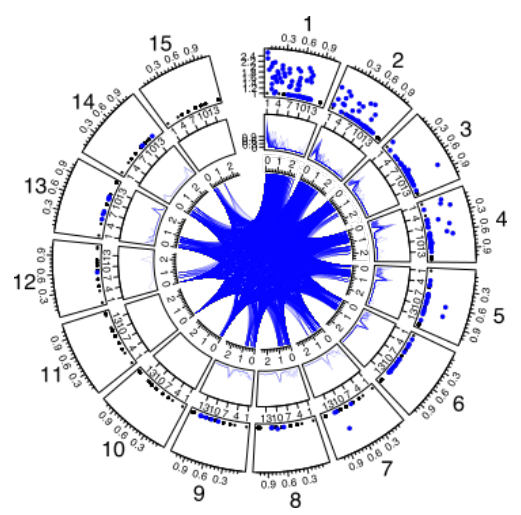

(d) MaF5 on PICEA-g

Fig. 5: MaF5 with 15 Objectives

\section{B. MaF5 Benchmark Problem}

This is a concave badly-scaled problem, with 15 objectives. The last objective is in the range $\left[0,2^{15}\right]$, the 14 th objective is in the range $\left[0,2^{14}\right]$ and so on. For this problem and instance, the NSGAIII algorithm, Fig. 5a, presented good convergence and dispersion. Most of the solutions obtained by the algorithms RVEA, Fig. 5b, and MOEA/DD, Fig. 5c, are located in the objectives 11 to 15 . The algorithm PICEAg, Fig. 5d, obtained the majority of its solutions distributed between objectives 1 to 9 and many of them with insufficient convergence. These results suggest that these methods are quite sensitive to badly-scaled problems though in different ways. Since NSGAIII relies on dominance relation, it is less sensitive to the scale of the objectives. On the other hand, HypE is strongly sensitive to scaling since it is guided by the hypervolume.

\section{MaF7 Benchmark Problem}

The MaF7 Benchmark Problem presents $2^{M-1}$ disconnected segments. The algorithms NSGAIII and RVEA, see Figs. 6a and 6c, present good convergence and dispersion. All solutions of the algorithms HypE and PICEA-g, Figs. 6b and $6 e$, are close to the objective 10 and the solutions obtained by the algorithm MOEA/DD are highly concentrated in sector 1, see Fig. 6d. This high concentration of solutions can be observed by the thick and well defined lines in tracks A and B of the graph.

The high concentration of solutions obtained by the MOEA/DD close to objective 1 can also be observed by the value of its $\mathrm{HV}$ metric, which presents a very different value from the values of this metric for the other algorithms. However, although this metric shows very close values for the other algorithms, the distribution of the solutions presents two very different patterns. While the NSGAIII and RVEA algorithms have good coverage of the Pareto Front, the solutions of the HypE and PICEA-g algorithms are concentrated in sector 10 with no solutions in the other sectors.

\section{MaF10 Benchmark Problem}

The MaF10 benchmark Problem has a complex and irregular shape containing concave and convex segments, and badlyscaled range of the Pareto Front. These characteristics can be observed by the irregular distribution of the points representing the Pareto Front in track $\mathrm{C}$. In this problem, the algorithms NSGAIII and RVEA, Figs. 7c and 7b, present good convergence and dispersion. The algorithm MOEA/DD, see Fig. 7c, presents good solutions close to the objectives 1 and 2, but few solutions in the other sectors. In a complementary way, the PICEA-g algorithm, see Fig. 7d, presents a set of solutions in sectors 3 to 9 but few solutions close to objectives 1 and 2 


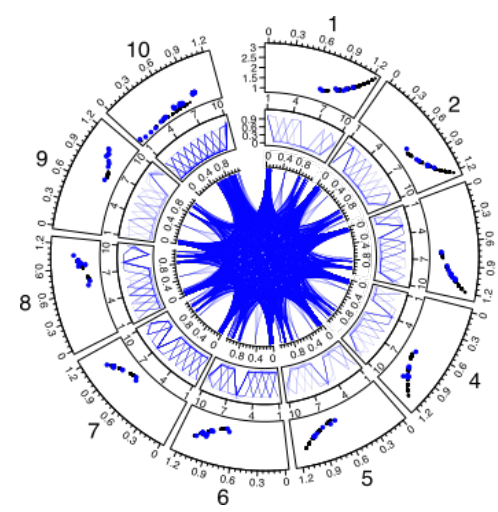

(a) MaF7 on NSGAIII

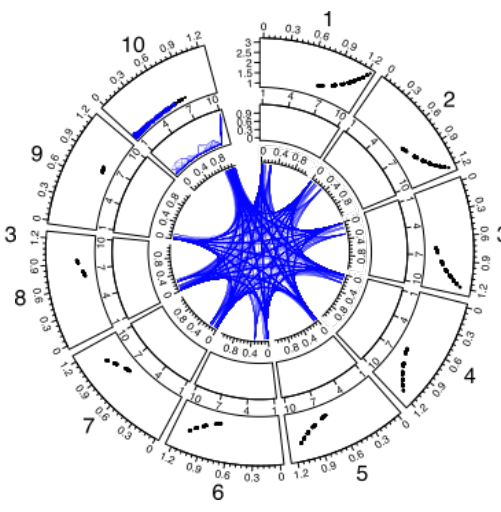

(b) MaF7 on HypE

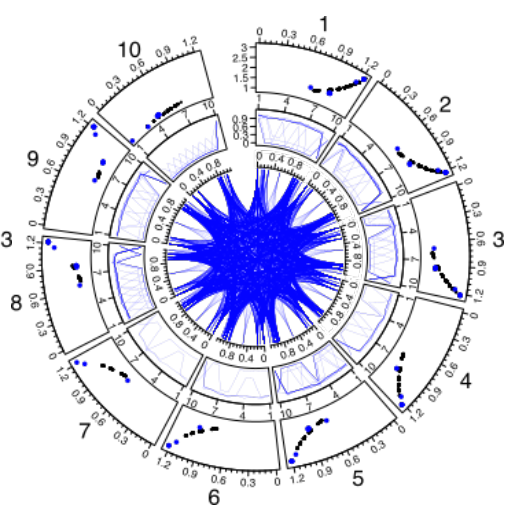

(c) MaF7 on RVEA

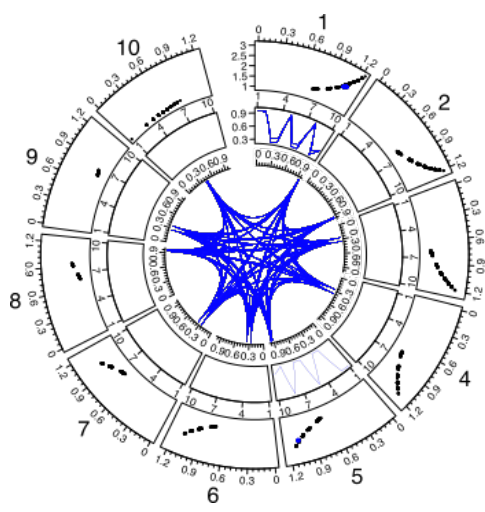

(d) MaF7 on MOEA/DD

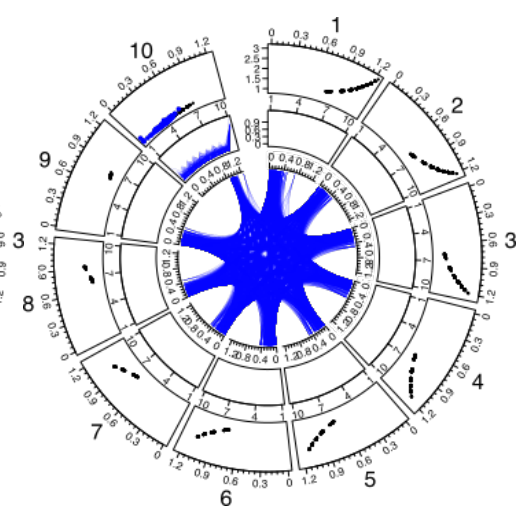

(e) MaF7 on PICEA-g

Fig. 6: MaF7 with 10 objectives

and no solution near objective 10. Despite the very different results regarding the distribution of the solutions obtained by the algorithms in this problem, they all have a very similar hypervolume metric.

\section{FinAl REMARKS}

This paper presented a new integrated visualization method able to present high quality information about the obtained solutions of many objective optimization problems. The grouped visualization method can display in a single graph more than one set of solutions, allowing the qualitative comparison of results of different MOEA in MaOPs. It is possible to see if there is the formation of clusters, those regions neglected by the algorithm, the spatial distribution of points as well as its proximity to the Pareto Front and the presence of harmony or conflict between objectives. This method can display the same amount of solutions of the traditional methods.

An important characteristic of the proposed method is that, for the same set of solutions, the information contained in one sector is independent of the information contained in the other sectors. In this way, for a high-dimensional optimization problem (more than 15 objectives, for example), the data can be divided into two or more circular graphs in order to avoid creating very narrow sectors.
Finally, this method of visualization and the results on the Benchmark Problems show the importance of the qualitative analysis of the data. An example of this is the MaF10 problem with 10 objectives, where the values of the HV are hard to interpret, but the graphs indicate a very different spatial distribution of the solutions obtained by each algorithm for that problem.

\section{REFERENCES}

[1] K. Deb, Multi-Objective Optimization Using Evolutionary Algorithms. New York, NY, USA: John Wiley \& Sons, Inc., 2001.

[2] A. Zhou, B.-Y. Qu, H. Li, S.-Z. Zhao, P. N. Suganthan, and Q. Zhang, "Multiobjective evolutionary algorithms: A survey of the state of the art," Swarm and Evolutionary Computation, vol. 1, no. 1, pp. 32-49, mar 2011.

[3] A. Trivedi, D. Srinivasan, K. Sanyal, and A. Ghosh, "A survey of multiobjective evolutionary algorithms based on decomposition," IEEE Transactions on Evolutionary Computation, vol. 21, no. 3, pp. 440-462, June 2017.

[4] C. von Lücken, B. Barán, and C. Brizuela, "A survey on multi-objective evolutionary algorithms for manyobjective problems," Computational Optimization and Applications, vol. 58, no. 3, pp. 707-756, 2014. 


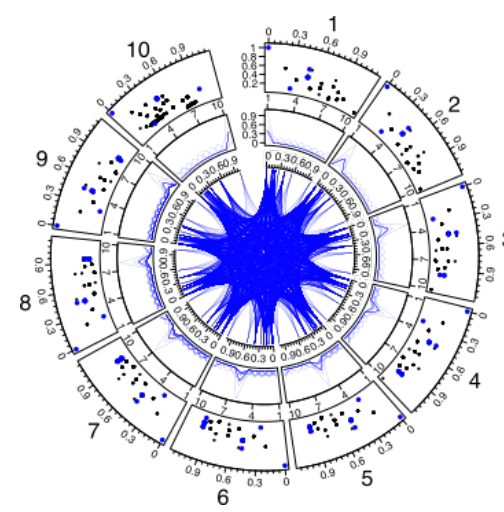

(a) MaF10 on NSGAIII

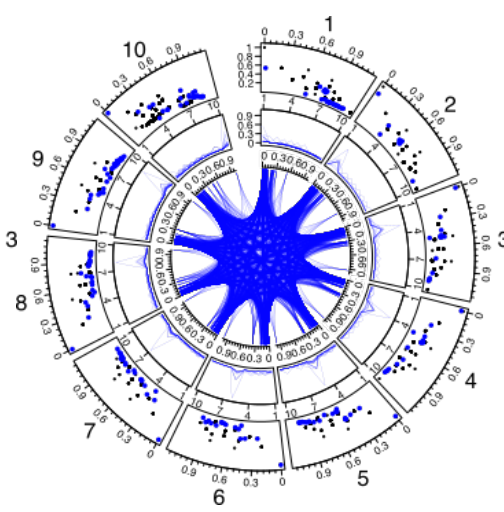

(b) MaF10 on RVEA

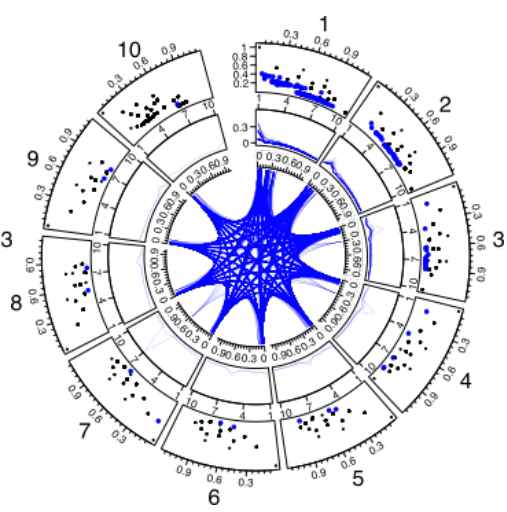

(c) $\mathrm{MaF} 10$ on MOEA/DD

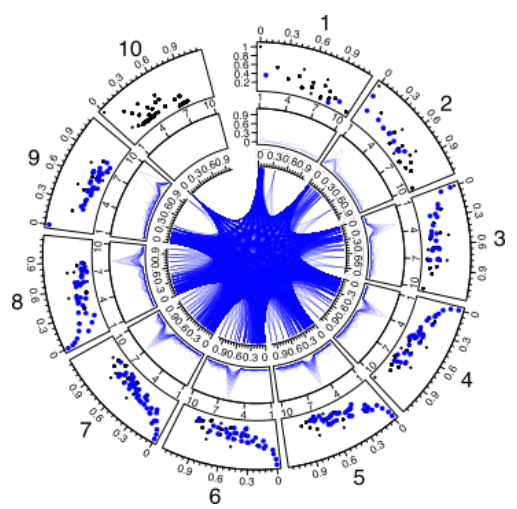

(d) MaF10 on PICEA-g

Fig. 7: MaF10 with 10 objectives

[5] B. Li, J. Li, K. Tang, and X. Yao, "Many-objective evolutionary algorithms: A survey," ACM Comput. Surv., vol. 48, no. 1, pp. 13:1-13:35, Sep. 2015. [Online]. Available: http://doi.acm.org/10.1145/2792984

[6] L. S. Batista, F. Campelo, F. G. Guimarães, and J. A. Ramírez, "A comparison of dominance criteria in many-objective optimization problems," in 2011 IEEE Congress of Evolutionary Computation (CEC), June 2011, pp. 2359-2366.

[7] A. R. de Freitas, P. J. Fleming, and F. G. Guimarães, "Aggregation trees for visualization and dimension reduction in many-objective optimization," Information Sciences, vol. 298, pp. 288-314, mar 2015.

[8] Z. He and G. G. Yen, "Visualization and performance metric in many-objective optimization," IEEE Transactions on Evolutionary Computation, vol. 20, no. 3, pp. 386-402, jun 2016.

[9] T. Tusar and B. Filipic, "Visualization of pareto front approximations in evolutionary multiobjective optimization: A critical review and the prosection method," IEEE Transactions on Evolutionary Computation, vol. 19, no. 2, pp. 225-245, apr 2015.

[10] C. M. Fonseca and P. J. Fleming, "Genetic Algorithms for Multiobjective Optimization: Formulation, Discussion and Generalization," 5th Int. Conf. Genetic Algo- rithms, pp. 416-423, 1993.

[11] M. Li, L. Zhen, and X. Yao, "How to read many-objective solution sets in parallel coordinates [educational forum]," IEEE Computational Intelligence Magazine, vol. 12, no. 4, pp. 88-100, nov 2017.

[12] A. Pryke, S. Mostaghim, and A. Nazemi, "Heatmap Visualization of Population Based Multi Objective Algorithms," in Evolutionary Multi-Criterion Optimization. Berlin, Heidelberg: Springer Berlin Heidelberg, 2007, pp. 361-375.

[13] D. J. Walker, R. M. Everson, and J. E. Fieldsend, "Visualisation and ordering of many-objective populations," in IEEE Congress on Evolutionary Computation. IEEE, jul 2010, pp. 1-8.

[14] _ _ "Visualizing mutually nondominating solution sets in many-objective optimization," IEEE Transactions on Evolutionary Computation, vol. 17, no. 2, pp. 165-184, 2013.

[15] A. R. R. Freitas, P. J. Fleming, and F. G. Guimaraes, "A non-parametric harmony-based objective reduction method for many-objective optimization," in 2013 IEEE International Conference on Systems, Man, and Cybernetics. IEEE, oct 2013, pp. 651-656.

[16] R. Silva, A. Salimi, M. Li, A. R. R. Freitas, F. G. Guimarães, and D. A. Lowther, "Visualization and anal- 
ysis of tradeoffs in many-objective optimization: A case study on the interior permanent magnet motor design," IEEE Transactions on Magnetics, vol. 52, no. 3, pp. 1-4, March 2016.

[17] R. H. Koochaksaraei, R. Enayatifar, and F. G. Guimarães, "A new visualization tool in many-objective optimization problems," in Lecture Notes in Computer Science. Springer International Publishing, 2016, pp. 213-224.

[18] R. H. Koochaksaraei, I. R. Meneghini, V. N. Coelho, and F. G. Guimarães, "A new visualization method in many-objective optimization with chord diagram and angular mapping," Knowledge-Based Systems, vol. 138, no. Supplement C, pp. 134 - 154, dec 2017.

[19] R. Cheng, M. Li, Y. Tian, X. Xiang, X. Zhang, S. Yang, Y. Jin, and X. Yao, "Benchmark functions for cec'2018 competition on many-objective optimization," Technical Report, 2018.

[20] R. Cheng, M. Li, Y. Tian, X. Zhang, S. Yang, Y. Jin, and $\mathrm{X}$. Yao, "A benchmark test suite for evolutionary manyobjective optimization," Complex \& Intelligent Systems, vol. 3, no. 1, pp. 67-81, mar 2017.

[21] K. Deb and H. Jain, "An evolutionary many-objective optimization algorithm using reference-point-based nondominated sorting approach, part i: Solving problems with box constraints," IEEE Transactions on Evolutionary Computation, vol. 18, no. 4, pp. 577-601, aug 2014.

[22] J. Bader and E. Zitzler, "HypE: An algorithm for fast hypervolume-based many-objective optimization," Evolutionary Computation, vol. 19, no. 1, pp. 45-76, mar 2011.

[23] R. Cheng, Y. Jin, M. Olhofer, and B. Sendhoff, "A reference vector guided evolutionary algorithm for manyobjective optimization," IEEE Transactions on Evolutionary Computation, vol. 20, no. 5, pp. 773-791, oct 2016.

[24] K. Li, K. Deb, Q. Zhang, and S. Kwong, "An evolutionary many-objective optimization algorithm based on dominance and decomposition," IEEE Transactions on Evolutionary Computation, vol. 19, no. 5, pp. 694-716, oct 2015.

[25] R. Wang, R. C. Purshouse, and P. J. Fleming, "Preference-inspired coevolutionary algorithms for many-objective optimization," IEEE Transactions on Evolutionary Computation, vol. 17, no. 4, pp. 474-494, aug 2013.

[26] Y. Tian, R. Cheng, X. Zhang, and Y. Jin, "PlatEMO: A MATLAB platform for evolutionary multi-objective optimization [educational forum]," IEEE Computational Intelligence Magazine, vol. 12, no. 4, pp. 73-87, nov 2017.

[27] Z. Gu, L. Gu, R. Eils, M. Schlesner, and B. Brors, "Circlize implements and enhances circular visualization in R," Bioinformatics, vol. 30, no. 19, pp. 2811-2812, 2014.

[28] M. Krzywinski, J. Schein, I. Birol, J. Connors, R. Gascoyne, D. Horsman, S. J. Jones, and M. A. Marra, "Circos: An information aesthetic for comparative genomics,"
Genome Research, vol. 19, no. 9, pp. 1639-1645, jun 2009.

[29] A. Zhou, Y. Jin, Q. Zhang, B. Sendhoff, and E. Tsang, "Combining model-based and genetics-based offspring generation for multi-objective optimization using a convergence criterion," in 2006 IEEE International Conference on Evolutionary Computation. IEEE, 2006, pp. 892-899.

[30] L. While, P. Hingston, L. Barone, and S. Huband, "A faster algorithm for calculating hypervolume," IEEE Transactions on Evolutionary Computation, vol. 10, no. 1, pp. 29-38, feb 2006. 